\title{
A Review on Salmonella Species Isolated from Human Blood and Its Antibiotic Susceptibility Pattern
}

\author{
Habtamu Tedila* Jifar Abera \\ College of Natural and Computational Science Department of Biology (Stream of Applied Microbiology), \\ Madda Walabu University, PO box 247, Bale Robe, Ethiopia
}

\begin{abstract}
Salmonellosis continues to be a major public health problem worldwide. It also contributes to negative economic impacts due to the cost of surveillance investigation, treatment and prevention of illness. As such, research on Salmonella has gained great interest and concern from scientists. The purpose of this review is to discuss the classification and nomenclature, characteristic, clinical manifestation, epidemiology, transmission vehicles, antibiotic susceptibility, Pathogenesis, Diagnosis, Treatment.
\end{abstract}

Keywords: Antibiotic susceptibility, Clinical manifestation, Epidemiology, Salmonella, Transmission.

DOI: $10.7176 / \mathrm{ALST} / 70-05$

\section{Introduction}

Salmonellosis is one of the most common infectious diseases of the world for both humans and animals. Food borne infections caused by Salmonella serotypes occurs at high frequency in industrialized nations and developing countries and is an important public health problem worldwide. Typhoid fever (enteric fever) caused by the bacterium Salmonella enteric serovar typhi is an endemic disease in the tropic and sub tropic. The disease is systemic and is often contracted by ingestion of food or water that is contaminated with the pathogen usually from a feco-oral source. The illness may be mild or severe but sometimes fatal. It is encountered worldwide but is primarily found in developing countries where sanitary conditions are poor (WHO, 2001). There are 16 million annual cases of typhoid fever, 1.3 billion cases of gastroenteritis and 3 million deaths worldwide due to Salmonella (Bhunia, 2008). It is among the most commonly isolated food borne pathogens associated with fresh fruits and vegetables. In recent years, the incidence of foodborne outbreaks caused by the contamination of fresh fruits and vegetables has increased and become a great concern in industrialized countries (Pui et al., 2011).

During the five-year period an average of 3,469 cases of salmonellosis were reported per annum (17.3 cases per 100,000 inhabitants) (Schaller, 1972). It was noted that cases of enteric fever, diagnosed clinically as typhoid fever, were almost three times the number of that of the other types of salmonellosis. This suggests a typhoid fever rate of approximately 4/100,000 inhabitants in the early 1960s. However, the diagnosis of typhoid fever was on clinical grounds and the only microbiological study at the time reported that less than $1 \%$ of over 700 stool specimens grew S. Typhi (Wallace, 1981).It was not until 1981 that a comprehensive study on invasive Salmonella in Ethiopia was conducted (Gedebou and Tassew,1981). The real situation of antibiotic resistance is also not clear since Salmonella are not routinely cultured and their resistance to antibiotics cannot be tested. As in a developed country, however, to control the spread of salmonellosis, surveillance for Salmonella serovar's and the assessment of antimicrobial susceptibility is essential. In Ethiopia there have been several studies conducted on salmonellosis which suggest an increase in the antibiotic resistance of Salmonella to commonly used antimicrobials in both the public health and veterinary sectors (Gedebou and Tassew, 1981). Presently a single published study was there to describe the different serovars of Salmonella circulating in Ethiopia (GebreYohannes , 2007).Circulating serovars may present a puplic health risk problem by transferring resistant Salmonella through food and other sources, therefore this presentation aimed to indicate the potential importance of food and other veterinary agents as a source of multiple antimicrobial resistant Salmonella for human infections and suggest the need for detailed epidemiological and molecular studies in food animals, food products and humans in Ethiopia.

\subsection{Characteristic of Salmonella}

Salmonella is a facultative anaerobe, gram negative, flagellated, rod-shaped bacterium which is about 2-3 x 0.4$0.6 \mu \mathrm{m}$ in size (Yousef and Carlstrom, 2003; Montville and Matthews, 2008). Salmonella causes a bacterial disease called salmonellosis, which is usually manifested by an acute enterocolitis, with sudden onset of headache, abdominal pain, diarrhea, nausea, and sometimes vomiting. Fever is frequently present in salmonellosis patients. Salmonella are non-fastidious as they can multiply under various environmental conditions outside the living hosts. They do not require sodium chloride for growth, but can grow in the presence of 0.4 to $4 \%$. Most Salmonella serotypes grow at temperature range of 5 to $47^{\circ} \mathrm{C}$ with optimum temperature of 35 to $37^{\circ} \mathrm{C}$ but some can grow at temperature as low as 2 to $4^{\circ} \mathrm{C}$ or as high as $54^{\circ} \mathrm{C}$ (Gray, 2005). They are sensitive to heat and often killed at temperature of $70^{\circ} \mathrm{C}$ or above. Salmonella grow in a pH range of 4 to 9 with the optimum between 6.5 and 7.5. They require high water activity (aw) between 0.99 and 0.94 (pure water 
aw $=1.0$ ) yet can survive at aw $<0.2$ such as in dried foods. Complete inhibition of growth occurs at temperatures $<7^{\circ} \mathrm{C}, \mathrm{pH}<3.8$ or water activity $<0.94$ (Hanes, 2003; Bhunia, 2008).

\subsection{Classification and nomenclature of salmonella}

Historically Salmonella had been named based on the original places of isolation such as Salmonella London and Salmonella Indian and also classified based on the host preferences. The first group includes host-restricted serotypes that infect only humans such as S. typhi. The second group includes host-adapted serotypes which are associated with one host species but can cause disease in other hosts serotypes such as S. Pullorum in avian. The third group includes the remaining serotypes typically, Salmonella enteritidis, Salmonella typhimurium and Salmonella heidelberg are the three most frequent serotypes recovered from humans each year (Boyen et al., 2008).

Kauffmann-White scheme classifies Salmonella according to three major antigenic determinants composed of flagellar $\mathrm{H}$ antigens, somatic $\mathrm{O}$ antigens and virulence, capsular $\mathrm{K}$ antigens. Therefore, further classification of serotypes is based on the antigenicity of the flagellar $\mathrm{H}$ antigens which are highly specific for Salmonella (Scherer and Miller, 2001). Bacteria can be classified based on phylogeny. A phylogenetic tree can be derived from the comparison with $16 \mathrm{~S}$ rRNA or other gene sequences. There are 2463 Salmonella serotypes which are now placed under 2 species due to the difference in 16S rRNA sequence analysis: Salmonella enterica (2443 serotypes) and Salmonella bongori (20 serotypes). The system is currently used by World Health Organization (WHO) Collaborating Centre, Centers for Disease Control and Prevention (CDC) and some other organizations.

\subsection{Epidemiology of salmonella}

Typhoid cases are stable with low numbers in developed countries, but non typhoidal salmonellosis has increased worldwide. Typhoid fever usually causes mortality in 5 to $30 \%$ of typhoid-infected individual in the developing world. A more accurate figure of salmonellosis is difficult to determine because normally only large outbreaks are investigated where as sporadic cases are under-reported. Data on Salmonellosis are scarce in many countries of Asia, Africa and South and Central America where only 1 to $10 \%$ of cases are reported (Portillo, 2004). Typhoid fever is endemic throughout Africa and Asia as well as persists in the Middle East, some eastern and southern European countries and central and South America. Typhoid incidence is endemic or as is typically low in the first few years of life peaking in school-aged children and young adults and then falling in middle age.

\subsubsection{Transmission of salmonella Infection}

Salmonella are widely distributed in nature and they survive well in a variety of foods. Poultry, eggs and dairy products are the most common vehicles of salmonellosis. In recent years, fresh products like fruits and vegetables have gained concern as vehicles of transmission where contamination can occur at multiple steps along the food chain (Bouchrif et al., 2009). However, Salmonella typhi and Salmonella paratyphi A do not have animal reservoir, therefore infection can be happened by eating the improperly handled food by infected individuals (Newell et al., 2010). The Salmonella cells can attach to food contact surfaces such as plastic cutting board which may develop into biofilm once attached and hence cause cross-contamination. Consequently, Salmonella can enter the food chain at any point from livestock feed, through food manufacturing, processing and retailing as well as catering and food preparation in the home (Wong et al., 2002). Disease surveillance reports frequently identify poultry (chickens, turkeys, geese and ducks) as the main vehicles in the salmonellosis outbrea

\subsubsection{Pathogenesis of salmonella}

Usually, human hosts ingest $S$. typhi with contaminated water or food(Levine, 2001).After ingestion, $S$. typhi passes through the upper gastrointestinal tract to the small intestine where it attaches to the tips of the villi, probably via cystic fibrosis trans membrane conductance regulator (CFTR)-receptor located there and either invades the intestinal mucosa directly or multiplies several days before invading, "After invasion, typhoid organisms reach the lamina propria and via the "M cells" of the intestinal Peyer's patches (PP) migrate into mesenteric lymph nodes where they multiply (Everest,2001). Bacteria released into the circulation via the thoracic duct disseminate widely (transient primary bacteremia) before being taken up by macrophages lining the sinusoidal walls of the liver, spleen, and bone marrow. The organisms can replicate at these locations and the reentry of bacteria into the blood stream (secondary bacteremia) marks the on set of the clinical disease (Hoffman, 1991). After a relatively sustained bacteremia typhoid organisms are removed from blood by the liver and excreted via biliary passage to lead to re-infection of the intestinal tract (second exposure of PP to $S$. typhi). At the sites of localization of $S$. typhi, the endotoxin of $S$. typhi induces macrophages to produce an array of cytokines, including tumor necrosis factor (TNF) and interferon, and various arachidonic acid metabolites. Cytokines alone, when acting locally at the sites of their production or when disseminated via the blood stream, can mediate the development of fever, intestinal necrosis, hepatic dysfunction, pneumonitis, thrombosis , vascular instability leading to shock, bone marrow depression, and altered consciousness (Newton and Krishna,2002 ). 


\subsection{Clinical manifestation of salmonella Infection}

In human disease, the clinical pattern of salmonellosis can be divided into four disease patterns namely enteric fever, gastroenteritis, bacteremia and other complications of non typhoidal salmonellosis as well as chronic carrier state. Enteric fever Salmonella typhi causes typhoid fever where as Paratyphoid A, B and C cause paratyphoid fever with symptoms which are milder and mortality rate that is lower for the latter (Threlfall Jand Ward, 1999). Typhoid encephalopathy, often accompanied by shock, is associated with high mortality. Slight gastrointestinal bleeding can be resolved without blood transfusion but in 1 to $2 \%$ of cases can be fatal if a large vessel is involved. Intestinal perforation may present with abdominal pain, rising pulse and falling blood pressure in sick people. Hence, it is very serious in 1 to 3\% of hospitalized patients (Hu and Kopecko, 2003; Parry, 2006).Gastroenteritis Non-typhoidal salmonellosis or enterocolitis is caused by at least 150 Salmonella serotypes with Salmonella typhimurium and Salmonella enteritidis being the most common serotypes. Bacteremia caused by Salmonella should be taken into account in cases of fever of unknown origin. Patients with bacteremia and other complications should be treated with antibiotics (Scherer and Miller, 2001; Hanes, 2003).Chronic carrier state Salmonellosis can be spread by chronic carriers who potentially infect many individuals, especially those who work in food-related industries (Parry, 2006).

\subsection{Diagnosis}

The clinical suspicion of typhoid fever must be confirmed by appropriate laboratory investigations. Cultures of blood and bone marrow aspirate can provide the definitive diagnosis of typhoid fever (Punjabi, 2000). The Widal test (Widal Sero- Diagnostic Test) is based on the fact that usually there is an increase in the titres of agglutinating antibodies against $\mathrm{O}$ and $\mathrm{H}$-antigens of $\mathrm{S}$. typhi during the course of typhoid fever. Recently, many new diagnostic tests have been developed for the detection of $S$. typhi antibodies, its antigen or DNA (Hashimoto et al., 2009).used a nested polymerase chain reaction (PCR) based on the H1d-flagellin gene to detect $S$. typhi in blood. In stool samples, these tests may detect other Salmonella serovars with H1d-flagellin gene or antigen (Cardona-Castro et al., 2000). Detection of $S$. typhi antigen in urine samples is problematic due to intermittent excretion of $S$. typhi via urine (Chaicumpa et al., 2012).

\subsection{Prevention}

According to the Centers for Disease Control and Prevention (CDC),approximately one in four Americans may experience some form of food-borne illness each year, and prevention of food-borne infections is fairly complex (Mead et al.,1999).The major routes of transmission of typhoid fever are through drinking water or eating food contaminated with Salmonella typhi. Prevention is based on ensuring access to safe water and by promoting safe food handling practices. Health education is paramount to raise public awareness and induce behaviour change. In countries in which typhoid is endemic, the most important action is attention to safe drinking water and disposal of sewage and Mass vaccination with typhoid vaccine is also effective. Travellers to endemic areas should also take precautions with regard to hygiene but they must receive vaccination too. At the food animal industry level, there have been many approaches used to prevent and control salmonellosis, including improved biosecurity, vaccination, use of competitive exclusion products, and the introduction of novel immune potentiators. However, these practices have had limited success so far. Due to this reason, the use of antimicrobial chemotherapy has been implemented in order to treat and control Salmonellosis. This has led to increased antimicrobial resistance among several Salmonella enterica serovars (CDC-NARMS, 2007).

\subsection{Treatment}

Salmonellae are difficult to eradicate from the environment. However, because the major reservoir for human infection is poultry and livestock, reducing the number of Salmonellae harbored in these animals would significantly reduce human exposure. The rediscovery of oral rehydration therapy in the 1960s provided a simple way to prevent many of the deaths of diarrheal diseases in general. Where resistance is un common, the treatment of choice is a fluoroquinolone such as ciprofloxacin. Otherwise, a third-generation cephalosporin such as ceftriaxone or cefotaxime is the first choice. Cefixime is a suitable oral alternative. Typhoid fever, when properly treated, is not fatal in most cases. Antibiotics, such as ampicillin, chloramphenicol, trimethoprimsulfam ethoxazole, amoxicillin, and ciprofloxacin, have been commonly used to treat typhoid fever in microbiology. Treatment of the disease with antibiotics reduces the case-fatality rate to about $1 \%$. When untreated typhoid fever continues for three weeks to a month. Death occurs in $10 \%$ to $30 \%$ of untreated cases. In some communities, however, case-fatality rates may reach as high as 47\% (WHO, 2001).

\section{Antibiotic susceptibility}

The resistance of Salmonella to a single antibiotic was first reported in the early 1960s (Montville and Matthews, 2008). The first major epidemic of multidrug resistant $S$. typhi was reported in 1972 in Mexico (Levine, 2004). 
Since then, an increasing frequency of antibiotic resistance has been reported from all parts of the world, (Samantray, 2000).NARMS (National Antimicrobial Resistance Monitoring System) tests for the following 17 antimicrobial agents: amikacin, ampicillin, amoxicillin-clavulanic acid, cefoxitin, ceftiofur, ceftriaxone, cephalothin, chloramphenicol, ciprofloxacin, gentamicin, kanamycin, nalidixic acid, streptomycin, sulfamethoxazole, sulfisoxazole, tetracycline, and trimethoprim-sulfamethoxazole. NARMS data can provide useful information about patterns of emerging resistance, which in turn can guide mitigation efforts (CDCNARMS, 2007). The causative organism Salmonella typhi has rapidly gained resistance to antibiotics like ampicillin, ceftriaxone, and cotrimoxazole, and also to previously efficacious drugs like ciprofloxacin (Butt et al, 2003). The emergence of antimicrobial resistance, especially the multidrug resistance to ampicillin, chloramphenicol, and cotrimoxazole, has further complicated the treatment and management of enteric fever (Jesudason and John, 1992). So antibiotic susceptibility test (AST) has an important role in the treatment of typhoid fever (Bauer et al., 2006).

\section{Conclusion}

In Ethiopia, as in other developing countries, it is difficult to evaluate the burden of salmonellosis because of the limited scope of studies and lack of coordinated epidemiological surveillance systems. In addition, underreporting of cases and the presence of other diseases considered to be of high priority may have over shadowed the problem of Salmonellosis (Oosterom, 2010). In developing countries like Ethiopia the situation of antimicrobial resisitance is more complex and difficult. This is because Salmonellae and other major zoonotic bacterial pathogens are not routinely cultured and their resistance to commonly employed antimicrobials both in the puplic health and verterinary practices is rarely determined (Leegard et al 1996;Molla et al.,1999).Previous studies undertaken in Ethiopia indicated the presence of a high level of antimicrobial resistance in Salmonella isolated from humans(Gedebou and Tasew, 1981;Gebre, et al .,1987;Mache,et al.,1997;Alemayehu et al 2003).

Finally, there is a need of continuous surveillance and sharing of antimicrobial susceptibility data for Salmonella among countries worldwide (de Oliveira, 2010) to ensure the effectiveness of control programmes. The report has further accentuated the growing concern about the presence of and the spread of multidrug resistant $S$. typhi there by underscoring the need for rational application of antibiotics and other necessary interventions that will help to control the menace of antibiotic resistance. Provision of potable water, accurate laboratory diagnosis, public education, and so forth, are, therefore, recommended. Surveillance programs to monitor antimicrobial resistance patterns in other parts of the state and the entire country in general are also recommended. Further Public health authorities should now devise ways of using the currently available improved typhoid vaccines, in large-scale nursery-based and school-based immunization programmed, and should monitor their public health impact.

\section{Reference}

Alemayeu D.molla and Muckle A (2003).Prevalence and antimicrobial resistance pattern of Salmonella isolates of apparently healthy slaughtered cattle in Ethiopia. Trop anl Hlth Prod. (In press).

Bauer, A.W., Kirby, W.M.M., Sherris J,C., Turk, M. (2006). Antibiotic susceptiblity testing by a standard single disc diffusion method. AM. J.Clin. Pathol.45.493-496.

Bhunia, A. K. (2008). Foodborne microbial pathogens: Mechanisms and pathogenesis. United States of America: Springer Science + Business Media, LLC.

Bouchrif, B., P aglietti, B., Murgia, M., Piana, A., Cohen, N., Ennaji, M., Rubino, S. and Timinouni, M. (2009). Prevalence and antibiotic-resistance of Salmonella isolated from food in Morocco. Journal of Infection in Developing Countries 28(3): 35-40

Boyen, F.,Haesebrouck, F., Maes, D., Van Immerseel, F., Ducatelle, R. and Pasmans, F. (2008). Non-typhoidal Salmonella infections in pigs: A closer look at epidemiology, and control.Veterinary Microbiology 130(12): 1-19. doi:10.1016/j.vetmic.2007.12.017

Butt, R. N. Ahmad, A. Mahmood, and S. Zaidi. (2003). "Ciprofloxacin treatment failure in typhoid fever case, Pakistan, EmergingInfectious Diseases, vol. 9, no. 12, pp. 1621-1622.

Cardona-Castro N, Gotuzzo E, Rodriguez M, (2000). Clinical application of a dot blot test for the diagnosis of enteric fever due to Salmonella enterica serovar Typhi inpatients with typhoid fever from Columbia and Peru. Clin Diag Immunol; 7:312-13.

CDC- NARMS. (2007)."National Antimicrobial Resistance Monitoring System.(NARMS): Enteric Bacteria." Retrieved June 14, 2007, fromhttp://www.cdc.gov/narms/

Chaicumpa W, Rungkunaporn T, Burr D, (2012). Diagnosis of typhoid fever by detection of Salmonella typhi antigen in urine. J Clin Microbiol; 30:2513-15.Christie AB. Typhoid and paratyphoid fevers. In: Infectious Diseases.

de Oliveira, F. A., Pasqualotto, A. P., da Silva, W. P. and Tondo, E. C.( 2010). Characterization of Salmonella enteritidis isolated from human samples. Food Research International, In Press, Corrected Proof. 
doi:10.1016/j.foodres.09.040

Everest P, Wain J, Robersts M. (2001). The molecular mechanisms of severe typhoid fever. Trends Microbiol; 9: 316-20.

Gebre-Yohannes A, Mamo K and Wolde H. (1987). R- Factor mediated multi drug resistance in Salmonella typhimurium isolates. Ethiop Med J .25:53.

Gebre-Yohannes A. (2007). Salmonella from Ethiopia: prevalent species and their susceptibility to drugs. Ethiop Med J 23: 97-102.

Gedebou M. and Tassew A. (1981). Antimicrobial Susceptibility patterns and R-factors among Salmonella and Shigella isolates. Ethio Med 18:7-14.

Gray, J. T. and Fedorka-Cray, P. J. (2005). Salmonella. In Cliver, D. and Riemann, H. P. (Eds.). Foodborne diseases, p. 55-68. San Diego: Academic Press

Hanes, D. (2003). International hand book of foodborne pathogens, p. 137-149. New York: Marcel Dekker, Inc

Hashimoto Y, Itho Y, Fujinaga Y. (2009). Development of nested PCR based on the Via B sequence to detect Salmonella typhi. J Clin Microbiol; 33: 775-77.

Hoffman SL. (1991). Typhoid fever. In: Strickland GT, (Ed). Hunter's Tropical Medicine. Seven tends. Philadelphia, WB Saunders, pp. $344-58$.

Hu, L. and Kopecko, D. J. (2003). Typhoid Salmonella. In Millotis,M. D. and Bier, J. W. (Eds.). International handbook of food born epathogens, p. 151-165. New York: Marcel Dekker, Inc.

Jesudason and T. J. John. (1992). “Plasmid mediated multi drug resistance in Salmonella typhi,” Indian Journal of Medical Research, vol. 95, pp. 66-67.

Leegard T.M., Van gestle M H., Petit PLC., van D e Klundert JAM.(1996). Antibiotic resistance mechanism in salmonella species causing bacteraemia in Malawi and Kenya.APMIS.104:302306.

Levine MM. (2001). Typhoid fever. In: Plotkin SA, Orenstein WA, (eds).Vaccine. Third Edn. Philadelphia, WB Saunders, pp. 781-814.

Mache A, Mengistu y and Cowley S. (1997). Salmonella sero groups identified from adult diarrheal outpatients in Addis Ababa, Ethiopia : Antibiotic resistance and plasmid profile analysis .East Afr med J.74:183-187.

Mead, P., Slutsker, L., Dietz, V., McCaig, L., Bresee, J., Shapiro, C., Griffin, P., Tauxe, R. (1999). "Food-related illness and death in the United States." EmergingInfectious Diseases 5(5): 607-625.

Molla B, Kleer J and Sinell H.J.(1999). Antibiotic resistance pattern of food borne Salmonella isolates in Addis Ababa .berl Munch Tierarztl Wschr .122:41-43.

Montville, T. J. Matthews, K. R. (2008). Food microbiology: An introduction (2nd ed.. United States of America: ASM Press, Washington.

Newell, D. G., Koopmans, M., Verhoef, L., Duizer, E., Aidara-Kane, A., S prong, H., Giessen, J. v. d. and Kruse, H. (2010). Food-borne diseases-the challenges of 20 years ago still persist while new ones continue to emerge. International Journal of Food Microbiology 139: S3-S15. doi:10.1016/j.ijfoodmicro.2010.01.021.

Newton CR, Krishna S. (2002). Severe falciparum malaria in children: current understanding of pathob physiology and supportive treatment.Pharmacol Ther; 79: 1-53.

Oosterom J. (2010). Epidemiological studies and proposed preventive measures in the fight against human salmonellosis. Int J Food Microbiol 12: 41-51

Parry, C. M. (2006). Epidemiological and clinical aspects of human typhoid fever. In Matroeni, P. and Maskell, D. (Eds.). Salmonella infections: Clinical, immunological and molecular aspects, p. 1-18. New York: Cambridge University Press

Portillo, F.G. (2004). Molecularandcellularbiology of Salmonella pathogenesis. In Cary, J. W. and Bhatnagar, D. (Eds.), Microbial foodborne diseases: Mechanisms of pathogenesis and toxin synthesis, p. 3-34). United States of America: Technomic Publishing Company, Inc.

Pui, C. F., Wong, W. C., Chai, L. C., Lee, H. Y., Tang, J. Y. H., Noorlis, A., Farinazleen, M. G., Cheah, Y. K. and Son, R. (2011). Bio film formation by Salmonella Typhi and Salmonella Typhimurium on plastic cutting board and its transfer to dragon fruit. International Food Research Journal 18: 31-38

Punjabi NH. (2000). Typhoid fever. In: Conn, HF (ed).Current Therapy. Latest Approved Methods of Treatment for the Practising Physicians. Philadelphia, WB Saunders, pp. 161-65.

Samantray SK. (2000). Typhoid fever resistant to furazolidine, Ampicillin, chloramphenicol and cotrimoxazole. Indian J Med Sci; 33; 1-3.

Schaller, KF .(1972). Geo medical monograph Series 3, Ethiopia: Typhoid Fever, Paratyphoid Fever, and other Salmonellosis 109.

Scherer, C. A. and Miller, S. I. (2001). Molecular Salmonellae. In b Groisman. E. A. (Ed.). Principles of bacterial pathogenesis, p. 265- 316. United States of America: Academic Press.

Threlfall J, Ward L, Old D. (1999). Changing the nomenclature of Salmonella. Comm Dis Publ Hlth ; 2(3):156-7

Wallace, CK . (1981). Approach to enteric infections. Ethiopian Medical Journal 15: 29-36. S1369-5274 
(03)00028-6.

WHO World Health Organization.(2001). Global Salmonella Surveillance, Customize report. Available at $h t t p:==$ thor.dfvf.dk=pls= portal=GSS.country_data set_rep.show_parms $($ accessed10=20=200). Geneva:

Wong, D. M. A., L. F., Hald, T., wolf, P. J. v. d. and Swanenburg, M. (2002). Epidemiology and control measures for Salmonella in pigs and pork. Livestock Production Science 76(3): 215-222. Doi:10. 1016/S0301-6226(02)00121-5.

Yousef, A. E. and Carlstrom, C.(2003). Salmonella. In Yousef, A. E and, C. ( Eds). Food microbiology: A laboratory manual, p. 167-205. New Jersey: John Wiley \& Sons. 